\title{
Novel Soft-Switching Converter with Magnetic Amplifiers
}

\author{
Milivoje Brkovic ${ }^{+}$, Andzrej Pietkiewicz ${ }^{*}$, and Slobodan Cuk ${ }^{+}$
}

${ }^{+}$CALTECH, Power Electronicsa Group

EE 116-81, 1201 E. California Blvd.

Pasadena, CA 91125

Email: brka@cco.caltech.edu

Abstract - A novel half-bridge DC-to-Dc converter combines the functions of loss-less soft-switching and secondary side output voltage regulation at constant switching frequency. This was made possible by using magnetic amplifiers in series with the rectifier diodes. The primary side switches are driven at constant frequency and $50 \%$ duty ratios with small dead-time and the output voltage is regulated by PWM control of the magnetic amplifiers on the secondary side. Experimental results obtained on $200 \mathrm{~W}-48 \mathrm{~V}$ prototype operated at $200 \mathrm{kHz}$ confirm these features.

\section{Introduction}

In recent years, soft-switching converters have received growing interest in high-frequency power conversion due to zero-voltage switching (ZVS) and low conduction losses with constant frequency, pulse-width-modulated (PWM) control characteristics. A large number of recent papers [2-10] reflect new interest in the use of constant frequency resonant-transition converters which combine resonant methods for low loss switching with PWM for control

The switching losses in the switches are, in general, minimized by providing bi-directional current source to charge and discharge capacitance across the current bi-directional switch (MOSFET). The phase-shifted fullbridge and saturable reactor assisted soff-switching converters have been intensively studied in recent years [3-9]. Soft-switching converters can be designed to operate efficiently at a given line and load conditions, but maintaining ZVS over wide variations in line and load requires a large circulating current that produces higher conduction losses. By using noncontrollable saturable inductors in the primary circuit, instead of resonan inductor, the range of soft-switching can be extended [5]. In ref. [8], saturable inductors are connected in series with rectifier diodes and controlled in order to reduce circulating current, but complex circuitry and feedback isolation are still required. While zero-voltage switching in the primary side switches could be provided, the turn-off losses in the rectifier diodes (due to reverse recovery current), and parasitic oscillations (caused by diode's junction capacitance and leakage inductance) require additional complex circuitry. Saturable reactors are very often used as snubbers for the output rectifiers and EMI noise suppressers [7], [11]. The alternative solution is to use active snubber with an additional penalty in cost and complexity [6].

In this paper we propose a novel half-bridge Dc-to-Dc converter with magnetic amplifiers [1] which provides soft-switching for wide load range from no-load to rated load for all switches, secondary side control circuitry, and constant frequency operation (Fig. 9). In Section 2, soft-switching mechanism is briefly reviewed on the example of the buck converter. A novel half-bridge converter is described in Section 3. After conventional half-bridge converter is briefly reviewed, description of the new converter and softswitching mechanism are given in Sections 3.1 and 3.2 , respectively. In Section 3.3, the proposed soft-switching mechanism is compared with other solutions. The output voltage regulation is explained in Section 3.4, and different realizations of the voltage bi-directional switches on the secondary side are discussed in Section 3.5. Extended range of soft-switching and simple solution for reduction of circulating current are given in Sections 3.6 and 3.7 respectively. Experimental results are given in Section 4.

This work was conducted under Power Electronics Program supported by grants from Rockwell International Inc., Apple Computer Inc., GEC Ferranti Defense Systems Ltd., Italtel, Genaral Electric Co., Day-Ray Products Inc., Southem California Edison, and Magne Tek.

0-7803-0891-3/93\$03.00 @1993IEEE

\author{
*ASCOM Energy Systems \\ Murtenstrasse 133, CH-3000 Bern 5 \\ Switzerland
}

\section{Review of Soft-Switching Mechanism}

Soft-switching mechanism is explained in the buck converter. In order to obtain zero-voltage switching at constant switching frequency, the active switch $Q I$ and rectifier diode $D I$ in the conventional buck converter from Fig. 1 . a are replaced with composite, current bi-directional switches $S I$ and $S 2$ (realized with MOSFET transistors in practice) as shown in Fig. 1.b. Capacitors across both switches are included in order to model either device parasitic capacitance or the externally added capacitor. Loss-less, softtransition (zero-voltage switching) in switches $S l$ and $S 2$ occurs during the time intervals when both switches are turned-OFF, and charge between their capacitors is exchanged in the resonant fashion. While the soft-transition from the top switch $S I$ to the bottom switch $S 2$ is inherently provided by the positive inductor current, transition from the bottom switch $S 2$ to the top switch $S 1$ requires negative current source to oppose the positive load current flowing through the bottom switch. The simplest solution is to design already existing output inductor $L$ such that its current is bi-directional with peak-to-peak magnitude greater than twice the dc load current, for all operating conditions of interest [2].

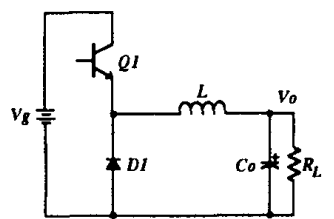

a)

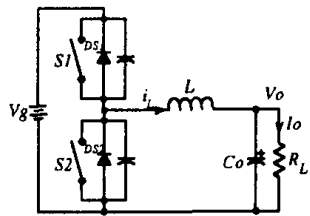

b)
Figure 1: a) Basic buck converter, and b) Soft-switching buck converter obtained by using composite switches $S 1$ and $S 2$.

Inductor current waveform during switching period and equivalent circuits of the converter during two different transition intervals are shown in Fig. 2. Duration of the transition intervals is assumed to be shor compared to the switching period $T_{s}$, so the inductor current is constant during these intervals as represented by constant dc current sources in the equivalent circuits in Fig. 2.b-c.

Transition from the top to the bottom switch (interval $t_{u}$ ) is initiated by turning-OFF the top switch so the constant, positive inductor current $I_{L p}=I_{L}+I_{o}$ (Fig. 2.b) linearly charges capacitor across the top switch (CSI) toward positive voltage and discharges capacitor across the bottom switch (CS2) toward negative voltage simultaneously as shown by dashed lines in the switch voltage waveforms. Once the voltage on the bottom switch reached zero, its diode (DS2) becomes forward biased and clamps the bottom switch voltage to zero, and consequently clamps the top switch voltage at bus voltage $V$. The bottom switch can now be turned-ON to zero voltage in loss-less manner, which completes the first transition interval.

Transition from the bottom to the top switch (interval $t_{12}$ ) starts by turningOFF the bottom switch $S 2$ carrying negative inductor current $I_{L n}=I_{L}-I_{o}$ (Fig. 2.a) which now provides opposite charge exchange between the switch capacitors. Now, the bottom switch capacitor $(C 2)$ charges toward positive voltage and the top switch capacitor discharges toward negative voltage as shown by dashed lines in Fig. 2.c. Once the voltage on the top switch reached the bus voltage $V_{e}$, its diode (DSI) becomes forward biased and clamps top 
switch voltage at zero and consequently the bottom switch voltage at $V_{g}$. The top switch $(S I)$ can now be turned-ON at zero-voltage in loss-less manner, which completes the second transition interval $t_{\mathrm{r} 2}$

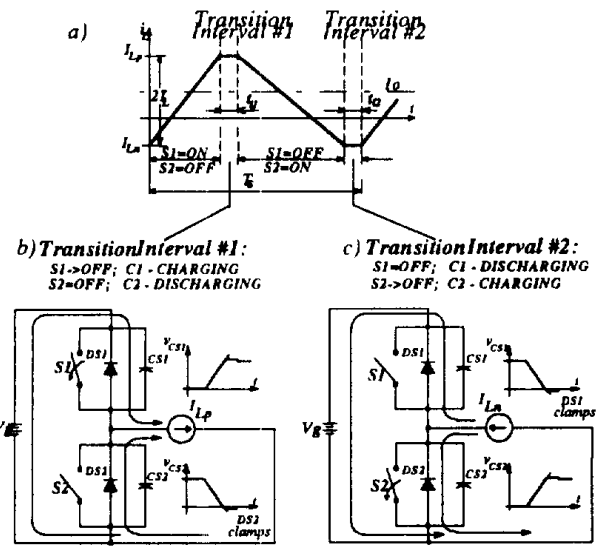

Figure 2: Soft-su'tching provided by: a) bi-directional current source, is explained using $b$ ) and c) equivalent circuits for two transition intervals.

Due to different inductor current levels $I_{L f}$ and $I_{L n}$, transition from the top to the bottom switch is always shorter than opposite transition from the bottom to the top switch particularly at high load currents. The amplitude of the inductor current $I_{L}$ has to be at least 3-4 times greater than the maximum load current in order to achieve sofi-switching for all operating conditions particularly at high switching frequency [2]. It results in increased conduction losses, increased core losses in the inductor, and requirement for additional output voltage filtening. Described soft-switching mechanism is inefficient at high load current levels, even though the loss-less switching can be achieved in very simple manner and voltage stresses on the switches are the same as in the parent PWM converter.

It is therefore, natural to search for the topologies which can provide softswitching by using DC output incuctor current with small or even no ripple current, instead of using high ripple, bipolar inductor current as in Fig. 2. Good candidates are transformer coupled converter topologies with full-wave rectification on their outputs, which naturally provide alternating current polarity to the primary switches before the beginning of transition intervals. In the following sections we describe the half-bridge converter which is the closest to the soft-switching buck converter from Fig. 2, but the same applies for the full-bridge and push-pull converters.

\section{A Novel Soft-Switching Converter}

Conventional half-bridge converter and idealized waveforms are shown in Fig. 3. Two equal capacitors $C l$ and $C 2$ are connected in series across the DC power supply $V_{g}$ to enable an artificial mid-point $P_{M}$ to be created. Primary side switches $S S^{g}$ and $S 2$ are driven alternatively during each switching period with duty ratio $D=\tau / T_{s}$. Full-wave rectification on the secondary side is provided by center-tapped secondary winding and rectifier diodes $D I$ and $D 2$. The output voltage $V_{o}$ is regulated by varying duty ratio $D$. The transformer's turns ratio is assumed to be $n=1$.

One can distinguish two successive operating states, active and passive during each half of the switching period $T_{s}$ (Fig. 3.b). During the active state $\tau$ the corresponding pair of the primary switch and rectifier diode $(S 1-D I$ or $S 2-D 2$ ) conduct simultaneously so the primary voltage and current have the same polarity and the power is delivered from the source to the load. Positive voltage of magnitude $V / 2$ is applied at the point $A$ and energy is stored in the output inductor $L$. Contrary, during the passive state $t_{d}$, both primary side switches are OFF but the rectifier diodes $(D I$ and $D 2)$ conduct inductor current simultaneously and short the transformer. As a consequence of that both primary voltage and current are zero and no power is delivered from the source to the load. Energy stored in the output inductor during active state is now released into the load through the rectifier diodes. This operating state is also called freewheeling state due to the nature of energy transfer.

Soft-switching is not possible because the transformer is shorted by the rectifier diodes just after the one of the primary side switches ( $S 1$ or $S 2$ ) is
turned-OFF so both switches, $S I$ and $S 2$ are connected instantaneously to the mid-point $P_{M}$ at voltage $V_{g} / 2$ and stay there during dead-time $\iota_{d}$. Capacitors across the primary switches, charged at the $V_{g} / 2$ during $t_{d}$, are therefore, discharged through the switches in lossy manner. It is therefore, necessary to prevent shorting of the transformer during freewheeling states. One solution. suggested in [9], is to use the transformer's magnetizing current for loss-less commutation of the primary side switches by adding freewheeling diode on the input of the output filter (point $A$ in Fig. 3.a) and providing that the resonant interval has ended before allowing the rectifier diode in the opposite secondary to conduct.

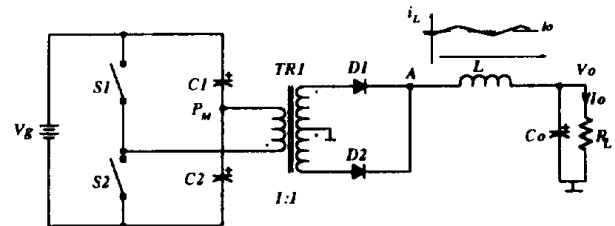

a)
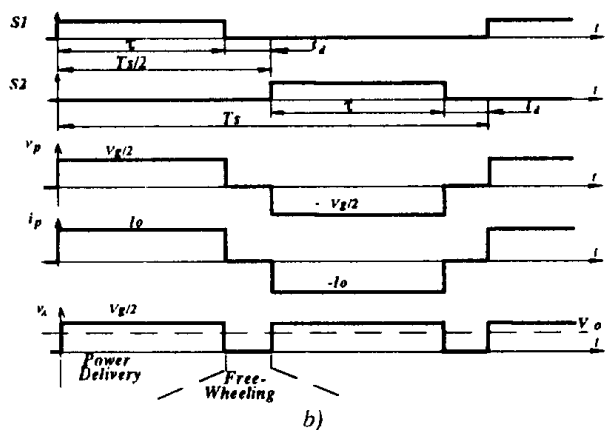

Figure 3: a) Conventional half-bridge converter and b) idealized waveforms.

We propose different mechanism for soft-switching which operates even with the ripple-free inductor current (DC current only) as described next.

\subsection{Circuit Description}

A novel soft-switching half-bridge converter is shown in Fig. 4. There are four main differences between proposed topology and conventional half-bridge converter from Fig. 3 :

a) Primary side switches $S I$ and $S 2$ are composite, current bi-directional switches (MOSFETs)

b) Primary side switches are driven alternatively at fixed duty ratio close to $50 \%$ with small dead-time $t_{d}$ (Fig. 5)

c) Rectifier diodes $D I$ and $D 2$ are replaced with controllable, voltage bidirectional switches $S 3$ and $S 4$, respectively

d) Output voltage is $P W M$ regulated by controlling the secondary side switches instead of the primary side switches

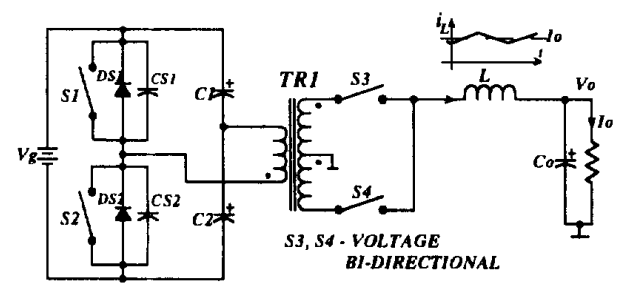

$$
\text { SI, S2 - CURRENT }
$$

Figure 4: A novel soft-switching half-bridge converter.

Both soft-switching of the primary side switches $S I$ and $S 2$, and output voltage regulation are obtained by using voltage bi-directional switches $S 3$ and $\$ 4$ with appropriate drives. 


\subsection{Soft-Switching Mechanism}

Idealized waveforms during a switching period and equivalent circuits of the converter during two transition intervals are shown in Fig. 5. The output inductor $L$ is assumed to be infinitely large so it is replaced with constant dc current source $I$ in the equivalent circuits in Fig. 5.b-c. Primary side switches, $S I$ and $S 2$, are driven alternately at fixed duty ratio close to $50 \%$ with small dead-time $t_{d}$, while the secondary side switches are driven alternately at exactly $50 \%$ duty ratio. During transition interval \#1 both primary switches are OFF but only one of the secondary side switches is forced to conduct the load current ( $S 3$ closed and $S 4$ open as in Fig. 5.b). Transformer is therefore, never shorted and primary current is maintained in the same direction during deadtime as it was before the primary switch is turned-OFF (dashed area in the primary current waveform in Fig. 5.a), so the soft-transition of the primary side switches is naturally provided as shown in Fig. 5.b. Primary current, being reflected DC load current $I$, naturally changes polarity between two transition intervals. Thus, soft-switching is also naturally provided during transition interval \#2 when $S 3$ is open and $S 4$ is closed as in Fig. 5.c.

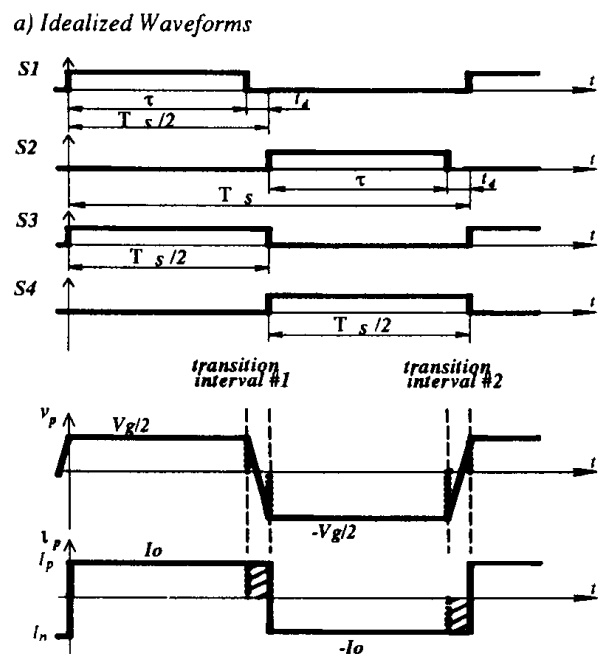

b) Transition Intenval \#I: SI $\rightarrow$ OFF; CSI - CHARGING

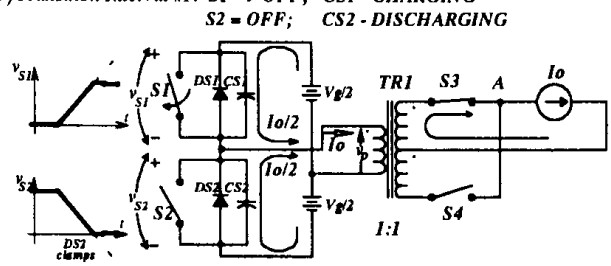

c) Transition Interval "2: SI =OFF; CSI - DISCHARGED S2 --> OFF; CS2-CHARGED

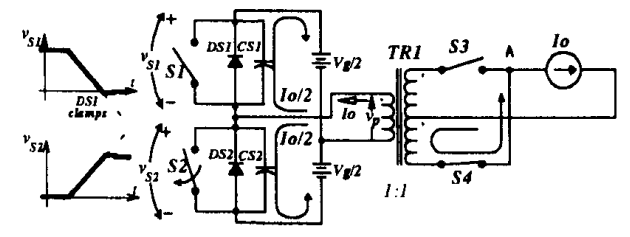

Figure 5: Soft-switching in the novel half-bridge converter (Fig. 4) is obtained by driving primary side switches $S 1$ and $S 2$ at $50 \%$ duty ratio with small and well controlled dead-time ${ }^{d} d$, and secondary side switches $S 3$ and

Transition between primary side switches is under the same scenario as described for the buck converter (Fig. 2) with only difference in the commutating current. Since in this case, the commutating current is reflected load current to the primary, both transitions are natural and equal (Fig. 5).

\subsection{Comparison of Soft Switching Mechanisms}

The main, and very important difference between two previously described soft-switching mechanisms is in the commutating current. Soft-switching mechanism in the half-bridge converter, even though similar to that in the buck converter, uses transformer's inherently bi-directional primary current (reflected DC load current) (Fig. 5) instead of the bipolar triangular inductor current with large ripple (Fig. 2). As a consequence of that, conduction losses in the switches, copper losses in the windings, and core losses in the output inductor, having (almost) DC current, are also reduced compared to the softswitching buck converter (Fig. 2). Moreover, duration of the transition intervals are the same compared to other solutions in which turn-ON and turnOFF transitions can differ an order of magnitude [3-8], unless some additional circuitry is added. Proposed soft-switching mechanism is also different from that suggested in [9] which uses the transformer's magnetizing current for softswitching. The main disadvantages of this solution are: a) magnetizing current needs to be relatively large to keep transition intervals short, b) freewheeling diode is essential for the soft-switching. Note that the proposed soft-switching half-bridge conventer operates without need for freewheeling diode.

\subsection{Output Voltage Regulation}

The main drawback of the described soft-switching half-bridge converter from Fig. 4 is that there is no output voltage regulation if the secondary side switches are driven as shown in Fig. 5.a. In order to provide the output voltage regulation it is necessary to vary duration of the interval during which voltage $V$ is negative, which occurs when the primary voltage and current are out of phase. The secondary side switches $S 3$ and $S 4$ are still driven out of phase with fixed $50 \%$ duty ratio but their drive signals are delayed by variable time $\theta$ (Fig, 6.c) with respect to original drive waveforms (Fig. 6.b)
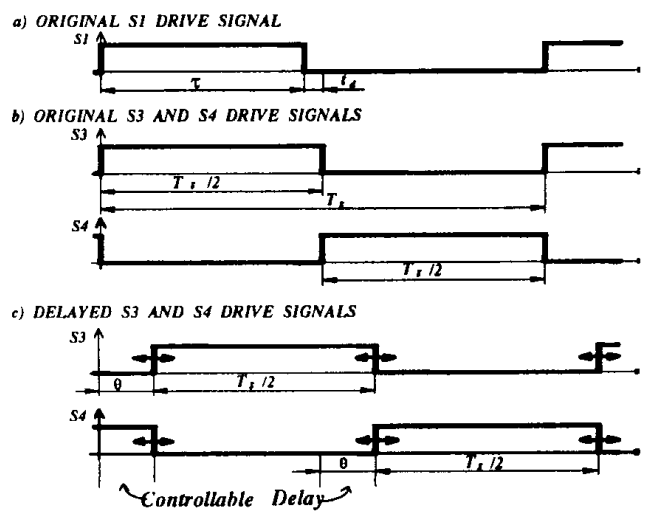

Figure 6: Output voltage regulation is provided by delaying drive signals for secondary side switches $S 3$ and S4 in respect to the drive signal for $S 1$

In addition to the active and passive states in the conventional converter (Fig. 3) there is soft-transition state $t_{d}$ in the new converter (Fig. 4) which separates the passive from the active state during half of a switching period as shown in Fig. 7. While the active states in new converter are the same as in conventional half-bridge converter, the behavior of the new converter is completely different during the passive, frewheeling state. During the active states $\tau-\theta$, the corresponding pair of the primary and secondary switches (SI-S3 or $S 2-S 4$ ) conduct simultaneously providing power deliver from the source to the load. Contrary, during the passive states $-\theta$, primary side switch conducts simultaneously with opposite secondary side switch (SI-S4 or $\$ 2-S 3$ ) making transformer's voltages and currents out of phase so that the load current circulates through the primary circuit and negative voltage is applied at the input of the output inductor (point $A$ ). Soft-transition states exist only between the active and the passive states, while transition from the passive to the active states are instantaneous. The output voltage regulation is therefore, achieved by varying duration of the passive states which corresponds to delay time $\theta$ in the drive signals of the secondary side switches $S 3$ and $S 4$. Duty ratio of the converter is defined as a portion of the switching period during which both voltages and currents in the transformer windings have the same polanty, i.e.: 

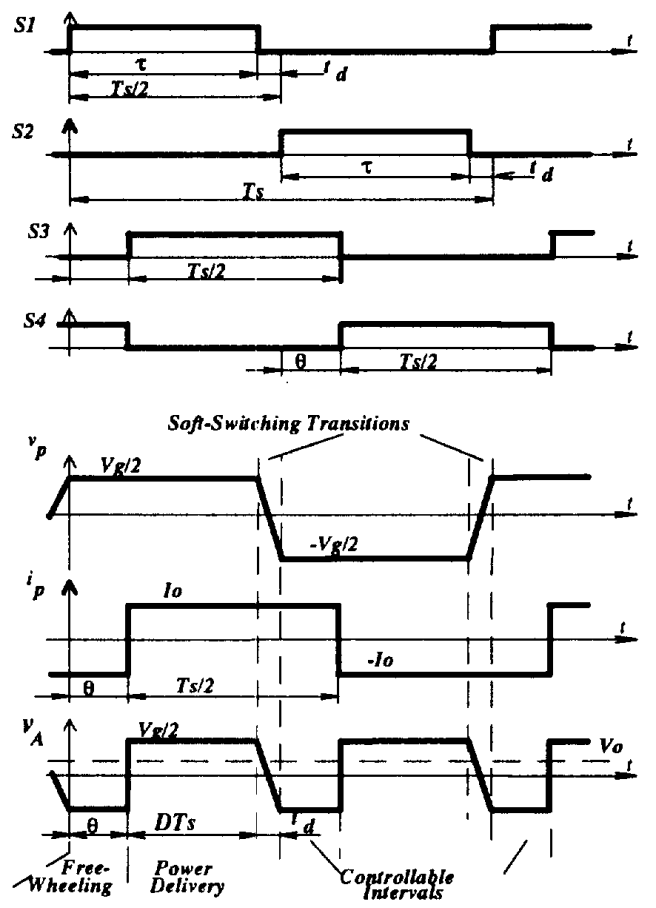

Figure 7: Idealized waveforms in the novel half-bridge converter (Fig. 5).

$$
D=2 \frac{\tau-\theta}{T_{s}}
$$

The output voltage is average value of the voltage $V_{A}$ at the input $A$, and in continuous conduction mode of operation $(C C M)$ is given by:

$$
V_{o}=V_{A}(2 D-1)=V_{g}(D-.5)
$$

\subsection{Switch Realization}

The primary current bi-directional switches, $S I$ and $S 2$, are realized in practice by the MOSFET transistors. On the other hand, there are three options for practical realization of the voltage bi-directional switches $S 3$ and $S 4$ on the secondary side of the converter as shown in Fig. 8.

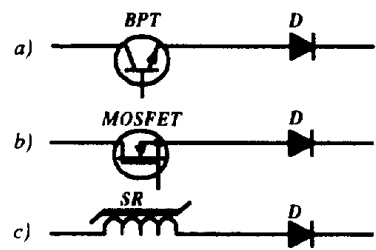

Figure 8: Three possible practical realizations of the voltage bi-directional switch.

Among them, realization with saturable reactor $S R$ in series with the diode $D$ (Fig. 8.c) is chosen due to several advantages such as:

a) there is no reverse-recovery problem during diode's turn-OFF so parasitic oscillations and voltage spikes in the diode voltage are significantly reduced

b) $E M I$ noise generation is reduced and overall converter efficiency is improved,

c) control of the salurable reactor is very simple [12] and does not require any synchronization with primary side switches nor complex control and drive circuits.
Soft-Switching of the Rectifier Diodes

Saturable reactors are widely used in series with rectifier diodes as magnetic snubbers and "spike-killers" [11]. Due to non-linear, square-loop magnetic characteristic, saturable reactor behaves as a switch. In saturation it behaves as a short circuit while out of saturation it represents a large inductance. By applying current of the opposite polarity, as is the case during diode's turn-OFF, saturable reactor runs out of the saturation at very small negative current and becomes large inductance which limits di/dt of the reverse current through the diode. By this action tum-off losses in the diode are minimized, and parasitic oscillations and voltage spikes are significantly reduced.

\subsection{Extended Range of Soft-Switching}

The common limitation for all zero-voltage switching topologies is the minimum load current at which zero-voltage switching is lost. Different techniques have been proposed for extending the range of zero-voltage switching but they require some additional resonant inductor [5], complex control circuitry [8] or active circuit [10].

The proposed converter can provide zero-voltage switching even at no load by increasing the dead-time $t_{d}$ in drive signals of the primary side switches $S I$ and $S 2$ (Fig. 5), and with proper design of the magnetizing inductance as shown in Fig. 11. Since converter operates in discontinuous conduction mode at light loads, reduction in the effective duty ratio due to increase in dead time will not affect output voltage regulation. Moreover, reset current and flux swing in the core of the magnetic amplifiers are simultaneously reduced due to reduction in the effective duty ratio. As a consequence of that, power consumed by the control circuit and core losses in the saturable reactors are reduced so the total efficiency of the converter is improved at light loads.

\subsection{Reduction of Circulating Current}

Circulating current in the primary circuit during freewheeling period $\theta$ (Fig. 7) results in higher conduction losses and needs to be minimized. By inserting freewheeling diode $D F W$ in series with small non-controllable saturable reactor SRFW (shown in dashed box in Fig. 9), it is possible to reduce circulating primary current as shown in Fig. 12. Conduction of the freewheeling diode $D F W$ is delayed so it starts to conduct after the resonant transition in the primary voltage is finished. In this manner, circulating current flows in the primary circuit only during transition interval $t_{d}$. Freewheeling diode is rated to much lower current than other two diodes $D R I$ and $D R 2$. This solution improves total efficiency of the converter with price paid for the additional diode and small saturable inductor.

\subsection{Multiple-Output and Other Topology Extensions}

The proposed topology is suitable for multiple-output extension since each output can be independently controlled by the magnetic amplifiers without affecting soft-switching operation of the converter. The problems inherent to the PWM half-bridge converter with mag-amps as a post-regulators [13] are eliminated since the transformer is never shorted.

The proposed concept for soft-switching is not limited to the half-bridge converter but it can be extended to other symmetrical topologies such as pushpull and full-bridge converters [1].

\section{Experimental Results}

In this section experimental results obtained on a $200 \mathrm{~W}, 48 \mathrm{~V}$ prototype operated at $200 \mathrm{kHz}$ from the $120 \mathrm{Vdc}$ are presented. Schematic of the novel soft-switching half-bridge converter is shown in Fig. 9. Circuit description is given in Section 4.1 and experimental waveforms are shown in Section 4.2.

\subsection{Circuit Description}

Power Stage

Primary side switches are realized with MOSFETs $Q 1$ and $Q 2$ while the secondary side, voltage bi-directional switches are realized with magnetic amplifiers $M A I$ and $M A 2$ in series with rectifier diodes $D I$ and $D 2$, respectively (Fig. 8). Isolation transformer $T R$ is designed with magnetizing 


\section{$T R$}

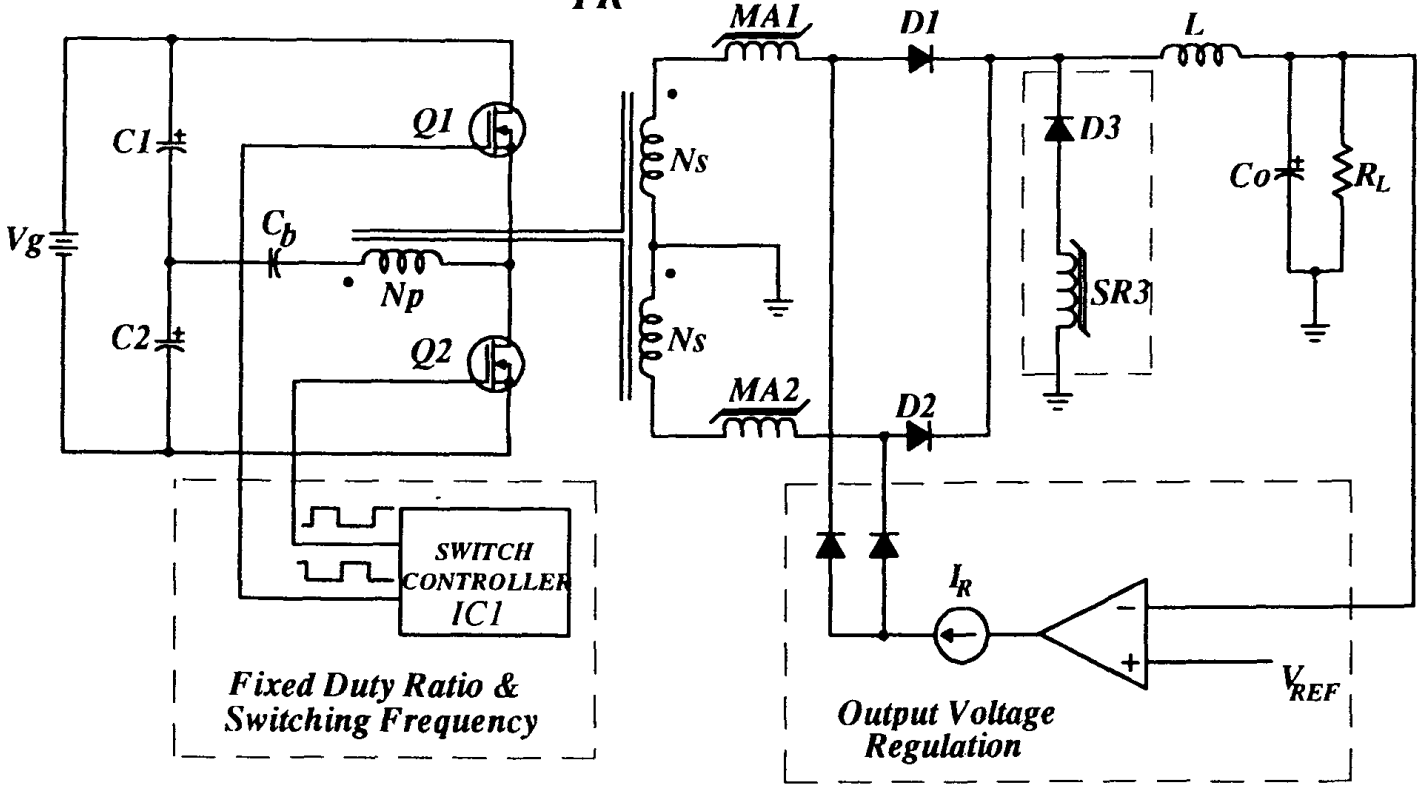

Figure 9: Soft-switching half-bridge converter with magnetic amplifiers. The primary switches $Q 1$ and $Q 2$ are complementary driven with fixed duty ratio close to 50\% at constant switching frequency. Output voltage is PWM regulated with magnetic amplifiers MAI and MA2.

inductance small enough to provide zero-voltage switching at light loads, or even no load as described in Section 3.6. There is no limitation for leakage inductance as in PWM parent converter, which together with small magnetizing inductance simplify transformer design. Diode $D 3$ and noncontrollable saturable inductor SR3, shown in dashed box, are added to reduce circulating current in the primary circuit after soft-transition is completed as described in Section 3.7. Output inductor is designed to have usually 20-30\% current ripple as in parent PWM converter.

\section{Control Circuit}

Control circuit on the primary side is realized with PWM chip with complementary outputs so the MOSFETs $Q 1$ and $Q 2$ are driven at constant switching frequency and $50 \%$ duty ratio with small dead-time. Voltage on the MOSFETs is sensed and used to delay turn-ON of the transistor if softtransition is not finished during dead-time $t$. In this way, dead-time is increased at light loads. Control circuit for the magnetic amplifiers is very simple and consist of error amplifier and voltage controlled current source. Output voltage is compared with the reference $V_{\text {ref }}$ in the error amplifier $E / A$. Output of the $E / A$ controls the reset current for the magnetic amplifiers, and as a consequence of that, their blocking capability. The very important advantage of secondary side control is that there is no need for isolation of the voltage feedback signal.

Components used in the prototype are listed bellow:

$Q 1, Q 2=I R F 640 ; D 1, D 2=$ MUR820; $D 3=$ MUR420; $I C I=U C 3846$; $M A 1, M A 2=50 \mathrm{~B} 66-1 \mathrm{E}$ (Magnetics); $S R 3=50 \mathrm{~B} 11-1 \mathrm{E}$ (Magnetics); $C 1, C 2=470 \mu \mathrm{F} ; C_{b}=2.2 \mu \mathrm{F} ; C_{o}=470 \mu \mathrm{F} ; L=42 \mu \mathrm{H} ; n=N_{p} / N_{s}=1$.

\subsection{Experimental Waveforms}

Salient waveforms obtained in $200 \mathrm{~W}, 48 \mathrm{~V}$ prototype operated at $200 \mathrm{kHz}$ are shown in Fig. 10. As can be seen primary voltage and current waveforms are trapezoidal and in a good agreement with idealized waveforms from Fig. 7.

Zero-voltage switching in the primary side switches is preserved even at no-load simply by increasing dead-time $t_{d}$ between drive signals for $Q 1$ and $Q^{2}$ as shown in Fig. 11. Transformer's magnetizing current is only $0.4 \mathrm{~A}_{\mathrm{p}-\mathrm{p}}$ and is enough to provide soft-transition in $1 \mu \mathrm{s}$. a)

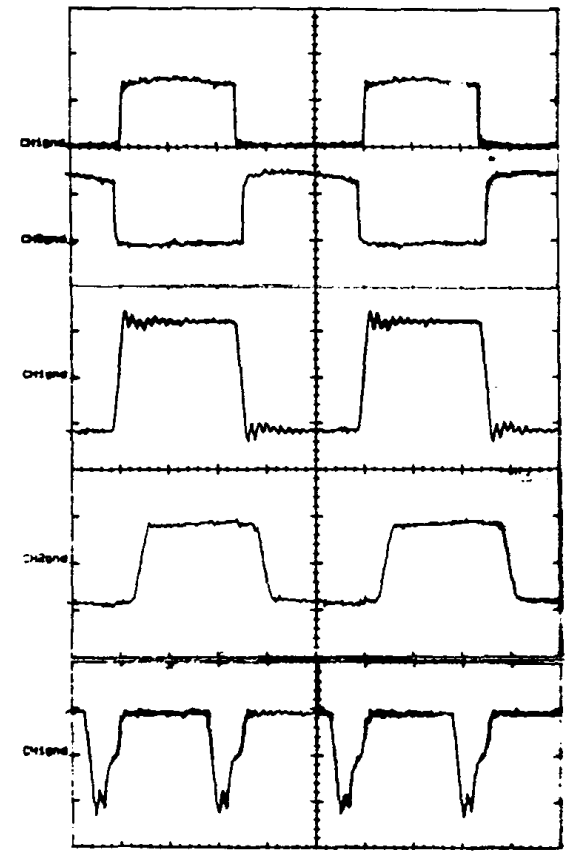

Figure 10: Salient waveforms obtained in $200 \mathrm{kHz}, 200 \mathrm{~W}-48 \mathrm{~V}$ prototype: a) Drive signals for $Q 1$ and $Q 2$; b) Primary voltage: 50Vldiv.; c) Primary current: 5 Aldiv.; d) Voltage at point A: 50V/div. Time: Ius/div.

Primary current and voltage waveforms are shown in Fig. 12.a for the converter without free-wheeling diode $D 3$ and non-controllable saturable inductor $S R 3$ (dashed box in Fig. 9). The same waveforms are then taken with these two components $D 3$ and $S R 3$ included (Fig. 12.b). As can be seen, 


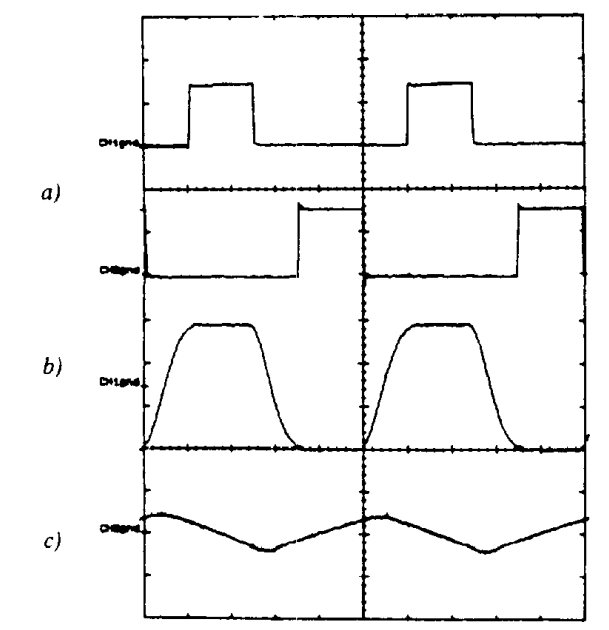

Figure 11: ZVS at no load condition is achieved simple by increasing dead time in drive signals: a) Drive signals; b) Primary voltage: 50V/div.; c) Primary current (Magnetizing current): ,4A/div. Time:1 usidiv.

primary current drops to zero after soft-transition is completed so the circulating current is indeed reduced.

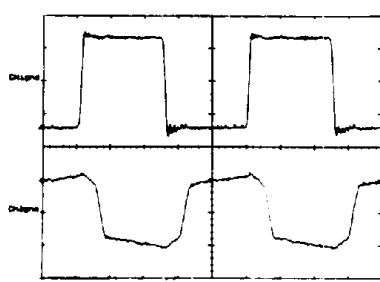

a)

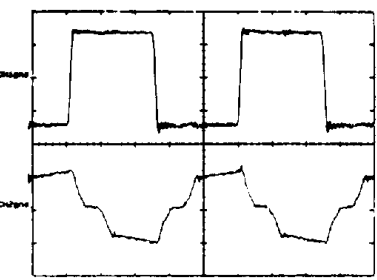

b)
Figure 12: Primary vollage and current waveforms: a) with no freewheeling diode in the output; and b) with freewheeling diode (Fig. 4). Voltage: 50Vldiv.; Current: 5Aldiv.: Time: Iusidiv.

\section{Conclusion}

A novel half-bridge Dc-Dc converter with magnetic amplifiers is presented which exhibits soft-switching in all switches and secondary side PWM output voltage regulation at constant switching frequency. Both soft-switching and the output voltage regulation are obtained by using voltage bi-directional switches on the secondary side realized with magnetic amplifiers in series with the rectifier diodes.

The proposed converter uses soft-switching mechanism different from the one used in buck converter which provides soft-switching from no-load to full load even with small output inductor ripple current and features symmetrical transition intervals.

Primary side switches, realized by MOSFET transistors, are alternatively driven at constant frequency and $50 \%$ duty ratio with small dead-time, while the secondary side switches are alternately driven at exactly $50 \%$ duty ratio with variable delay with respect to the primary switches.

Zero-voltage switching in the MOSFET transistors is naturally provided by reflected DC load current to the primary side which maintains the primary current in the same direction as before the MOSFET was turned-OFF. Zerocurrent switching of the rectifier diodes is provided by the action of the magnetic amplifiers due to their non-linear, square loop magnetic characleristic. The outpul voltage regulation is achieved by varying blocking interval of the magnetic amplifiers using control circuit on the secondary side, which eliminated the need for isolation of the feedback signal and resulted in a simple non-isolated control circuit.

The proposed novel soft-switching converter with magnetic amplifiers, described here for half-bridge converter, eliminates the key disadvantages of the conventional soft-switching converter topologies while keeping the simplicity of original pulse width-modulated $(P W M)$ converter.

\section{References}

[1] A. Pietkiewicz, and S. Cuk, "Soft-Switching Converters with Magnetic Amplifiers," CALTECH Patent Application, March 12. 1991.

[2] H. C. Martin, and D. W. Parsley. "Zero-Voltage Switching in High Frequency Power Converters Using Pulse Width Modulation," IEEE APEC Record, 1988, pp. 33-40.

[3] R. A. Fisher K. D. T. Neo and M. H. Kuo, "A 500kHz, 250W Dc-Dc Converter with Multiple Outputs Controlled by Phase-Shifted PWM and Magnetic Amplifiers," HFPC Conference Record, 1988, pp. 100-110

[4] R. Redl, N. O. Sokal, and L. Balogh, "A Novel Soft-Switching Full-Bridge DC/DC Converter: Analysis, Design Considerations, and Experimental Results at 1.5kW, 100kHz," IEEE PESC Record, 1990, pp. 162-172.

[5] G. Hua, F. C. Lee, and M. M. Jovanovic, "An Improved Zero-VoltageSwitched PWM Converter Using a Saturable Inductor," IEEE PESC Record, 1991, pp. 189-194.

[6] K. Harada and H. Sakamoto, "Switched Snubber for High-Frequency Switching," IEEE PESC Record, 1990, pp. 181-188.

[7] S. Hamada, Y. Manuama, and M. Nakaoka, "Saturable Reactor Assisted Soft-Switching Technique in PWM Dc-Dc Converters," IEEE APEC Record, 1992, pp. 93-100.

[8] M. M. Walters, and W. M. Polivka, "Extending the Range of Soft-Switching in Resonant-Transition Dc-Dc Converters," IEEE INTELEC Record, 1992. pp. 343-350.

[9] R. Farrington, M. M. Jovanovic, and F. C. Lee, "A New Family of Isolated Zero-Voltage-Switched Converters," IEEE PESC Record, 1991, pp. 209. 215.

[10] G. Hua, C. S. Leu, and F. C. Lee, "Novel 7ero-Voltage-Transition PWM Converters," IEEE PESC Record, 1992, pp. 55-61.

[11] O. Arakawa, T. Yamada, and R. Hiramatsu, "Magnetic Snubber Using Amorphous Saturable Reactor - Amorphous Beads," IEEE APEC Record, 1988, pp. 334-340.

[12] T. Koysshiki, and T. Ogata, "Design considerations in multiple-output dc converter with magnetic amplifiers," IEEE INTELEC Record, 1983, pp. 388-394.

[13] C. Jamerson, and D. Chen, "Magamp Post Regulators for Symmetrical Topologies with Emphasis on Half-Bridge Configuration," IEEE APEC Record, 1991, pp. 380-384. 